\section{SÉROPRÉVALENCE DE LA TOXOPLASMOSE DANS UNE POPULATION RURALE DU SUD-EST DU GABON}

BISVIGOU U.***, MICKOTO B.**, NGOUBANGOYE B.*, MAYI TSONGA S.**, AKUE J.P.* \& NKOGHE D.****

Monsieur,

La toxoplasmose est une anthropozoonose cosmopolite à Toxoplasma gondii, dont la prévalence est fonction des zones géo-climatiques et des habitudes alimentaires. Sa transmission à l'homme se fait par la consommation de viande peu cuite contenant des kystes du parasite, par voie transplacentaire ou par l'ingestion accidentelle d'oocytes provenant des fèces de chats, hôtes définitifs du parasite. Ces oocystes sont contenus dans les aliments, les eaux de boisson, le sol où se fait la maturation du parasite (Carme et al., 2006). Généralement bénigne et asymptomatique, conférant une immunité définitive, hormis dans les cas d'immunodépression, la toxoplasmose primaire peut se manifester par un syndrome mononucléosique fait de légère fièvre, myalgie, asthénie et de petites adénopathies non inflammatoires cervicales qui disparaissent spontanément après une semaine. Les formes graves, se rencontrent chez les nouveaux-nés et les immunodéprimés, notamment les séropositifs au VIH. Des formes sévères ont été observées chez des patients immunocompétents en Guyane française (Carme et al., 2006), avec la présence d'un cycle sylvatique.

Deux études menées au Gabon, entre 1978 et 1987 ont montré que la séroprévalence globale de la toxoplasmose était de 62,7 \% (Duong et al., 1992a et 1992b). Cette affection ne constitue pas isolément un problème de santé publique, mais elle suscite un intérêt particulier au cours des infections opportunistes du VIH/SIDA où elle est à l'origine de troubles neurologiques graves (Okome-Nkoumou et al., 2000; Okome-Nkoumou et al., 2006). Cet intérêt est motivé par la séroprévalence assez élevé du VIH, récemment estimée à 7,5\% au Gabon (Mintsa-Ndong et al., 2009). Les prévalences de la toxoplasmose au sein des animaux domestiques et des félidés sauvages sont également élevées (Ngoubangoye, 2007).

\footnotetext{
* Centre International de Recherches Médicales de Franceville, Gabon ${ }^{1}$. *** Faculté de Médecine et des Sciences de la Santé, Libreville, Gabon. **:* Ministère de la Santé et de l'Hygiène Publique, Libreville, Gabon. Correspondance : Jean-Paul Akue, BP 769, CIRMF, Franceville, Gabon. Tél. : +24106274162 - Fax : +24167 7295.

E-mail : jpakue@yahoo.fr

${ }^{1}$ Le Centre International de Recherches Médicales de Franceville (CIRMF) est supporté par le Gouvernement gabonais, Total Gabon et le Ministère français des Affaires étrangères.
}

Nous avons étudié le portage des anticorps dirigés contre Toxoplasma gondii dans une population rurale du sud-est du Gabon, afin d'en réévaluer localement la prévalence, le risque potentiel au cours de la grossesse et du SIDA.

Dienga est un village d'environ 2000 habitants, en zone de forêt équatoriale secondaire du sud-est du Gabon, où le Centre International de Recherches Médicales de Franceville (CIRMF) mène depuis 1990 diverses activités de recherche. Les sérums analysés proviennent de patients résidant dans ce village, prélevés entre juin 2006 et décembre 2007, pour divers protocoles de recherche, bilan prénatal ou à visée diagnostique, sans stratification ni sélection des individus. Les patients inclus n'avaient aucun signe évocateur d'une toxoplasmose. Des données sociodémographiques ont été récoltées et un consentement éclairé individuel a été obtenu avant tout prélèvement.

Les prélèvements ont été réalisés sur tube sec de type Vacutainer $^{\circledR}$. Les échantillons de sang prélevés ont été acheminés dans une glacière jusqu'à Franceville et conservés à $4^{\circ} \mathrm{C}$ en attendant les tests. Les dosages d'anticorps ont été été réalisés en utilisant le système automatisé Mini Vidas ${ }^{\circledR}$ de Biomérieux, combinant méthode immunoenzymatique sandwich en deux étapes et ELFA (Enzyme Linked Fluorescent Assay), selon les instructions du fabricant. Pour la toxoplasmose, nous avons utilisé le kit Vidas ${ }^{\circledR}$ Toxo IgM (TXM) pour les IgM et le kit Vidas ${ }^{\circledR}$ Toxo IgG II (TXG) pour les IgG. Les seuils de positivité retenus étaient de 0,65 de densité optique pour les IgM et de $8 \mathrm{UI} / \mathrm{ml}$ pour les d'IgG. Les sérologies VIH ont été été réalisées, en accord avec les patients, à l'aide du kit Vidas ${ }^{\circledR}$ HIV DUO Ultra (HIV5) et le seuil de positivité admis de 0,28 de densité optique. Les analyses statistiques ont été faites à l'aide du logiciel EPIINFO $2000^{\circledR}$, en utilisant le test de $\mathrm{khi}^{2}$ pour comparer les variables qualitatives, avec correction de Fisher pour des effectifs théoriques inférieurs à 5 , et le test t de Student pour les variables quantitatives. Le risque de première espèce admis était $\mathrm{a}<0,05$. Les variables étudiées étaient l'âge, le sexe, les taux d'IgM et d'IgG anti $T$. gondii, la sérologie HIV (positif ou négatif).

Les prélèvements analysés provenaient de 355 personnes, âgées de 1 à 85 ans; la moyenne d'âge était de 26 ans, le sex ratio de 0,68. La prévalence de positivité en IgG dans la population d'étude était de 75,5\% (IC $95 \%=[70,7-79,9])$. La séroprévalence au sein des femmes en âge de procréer (130 femmes âgées de 14 à 45 ans) était de 80,8 \% (IC $95 \%$ [73,4-87,6]). Parmi les 31 femmes enceintes qui ont participé à notre étude, 
deux étaient non immunisées contre la toxoplasmose; aucune séroconversion n'avait été observée au cours des contrôles mensuels, ni de signes de toxoplasmose congénitale chez les enfants nés au cours de la période d'étude. Dans notre population, l'acquisition de l'immunité était précoce, la séroprévalence atteignait de 57,4\% avant 15 ans et se maintenait autour de $80 \%$, au-delà de 15 ans (tableau I). Une croissance rapide de l'immunité a été observée dans les trois sous-classes d'âges des moins de 15 ans, où les taux étaient de 24,24\% (8/33) entre 1 et 5 ans, $61,5 \%(24 / 39)$ entre 6 et 10 ans et $83,3 \%(30 / 36)$ entre 11 et 14 ans (figure 1). La séroprévalence augmente significativement avec l'âge ( $\mathrm{p}<$ 0,001). Seuls deux enfants testés présentaient des IgM antitoxoplasmiques : une fille de 10 ans avec un taux d'IgG de $100 \mathrm{UI} / \mathrm{ml}$ et un garçon de 12 ans, négatif en IgG. Des tests de contrôle n'ont pu être réalisés. Enfin, la séroprévalence du VIH, chez 157 individus testés, était de 9,6\% (IC $95 \%=[5,4-15,3])$.

\begin{tabular}{lccc}
\hline $\begin{array}{c}\text { Classes } \\
\text { d'âges }\end{array}$ & $\begin{array}{c}\text { Positifs/testés (\%) } \\
(\mathbf{n}=\mathbf{3 5 5})\end{array}$ & IC $\mathbf{9 5} \%$ & $\mathbf{p}$ \\
\hline $1-14$ & $62 / 108(57,4)$ & {$[47,5-66,9]$} & Référence \\
$15-30$ & $114 / 142(80,3)$ & {$[72,8-86,5]$} & $\mathrm{p}<0,0001$ \\
$31-45$ & $53 / 59(89,8)$ & {$[79,2-96,2]$} & $\mathrm{p}<0,0001$ \\
$>45$ ans & $39 / 46(84,8)$ & {$[71,1-93,7]$} & $\mathrm{p}<0,001$ \\
\hline
\end{tabular}

Tableau I. - Répartition de la séroprévalence IgG de T. gondii en fonction des classes d'âge.

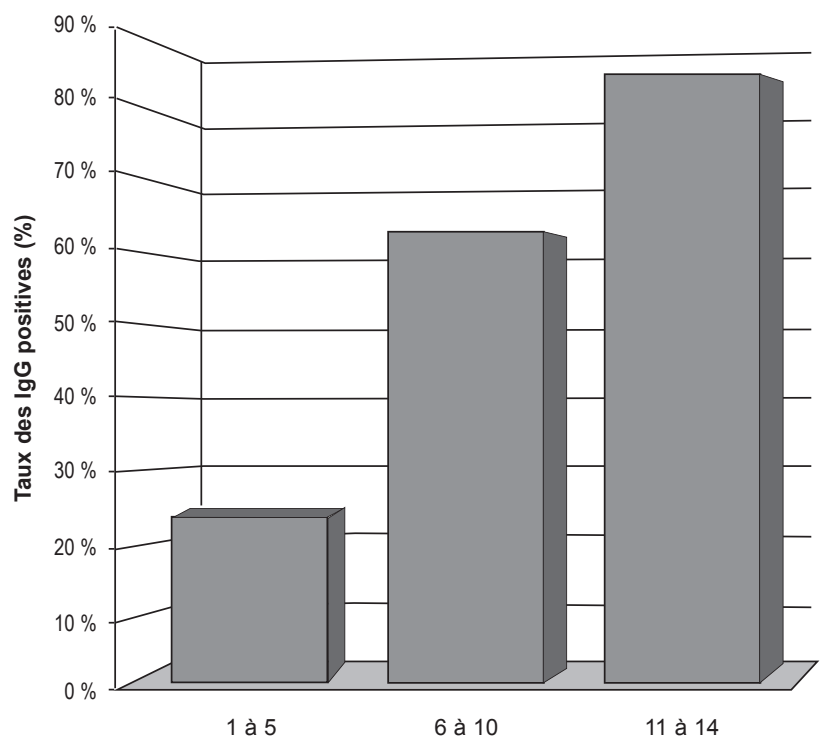

Fig. 1. - Prévalences des IgG antitoxoplasmiques observées chez les enfants de moins de 15 ans à Dienga.
La prévalence des IgG antitoxoplasmiques dans notre étude, réalisée dans une population rurale, était de 75,5\%. Elle n'a pas évolué en 20 ans, puisque des données relevées antérieurement dans une population identique (Duong et al., 1992a), et urbaine (Duong et al., 1992b) sont superposables. L'incidence et la transmission de cette maladie sont donc restées stables en zone de forêt. Nous constatons toutefois des différences de prévalence avec des zones de savanes gabonaises (Beauvais et al., 1978; Billiault et al., 1987) et d'autres pays de l'Afrique de l'ouest tels que le Nigéria (Uneke et al., 2007) et de l'Afrique de l'est comme l'Ethiopie (Negash et al., 2008).

Dans notre échantillon, la séroprévalence chez les enfants de moins de cinq ans était proche de celles observées à Libreville (Duong et al., 1992), en milieu urbain, et sur l'île de Sao Tomé et Principe qui dispose des mêmes conditions géoclimatiques (Fan et al., 2006). Ces éléments plaideraient pour une transmission précoce et stable dans la sous région. L'acquisition très précoce des anticorps, l'augmentation rapide des taux avec l'âge, et leur stabilité après 15 ans, évoqueraient un modèle épidémiologique tropical connu (Carme et al., 2006). Dans ce modèle, les conditions d'hygiène et climatiques étant favorables à la dissémination des sporozoïtes, les séroprévalences des adultes sont atteintes dès l'adolescence. Au Gabon, la viande, produit de la chasse, est toujours consommée bien cuite et constitue la principale source protéique des populations rurales. La contamination serait alors d'origine tellurique, par ingestion du parasite contenu dans le sol, à travers les aliments souillés. La forte prévalence observée chez les animaux domestiques, félidés compris, suggère que ces derniers joueraient un rôle de réservoir et source de propagation tellurique (Ngoubangoye, 2007).

Dans notre étude, $80 \%$ des femmes en âge de procréer étaient immunisées, traduisant une protection quasi complète de la population concernée. Le suivi des femmes enceintes non immunisées n'a déterminé aucune séroconversion, et aucun des nouveaux-nés n'a présenté des signes évocateurs de toxoplasmose.

La prévalence du VIH dans notre échantillon était de 9,6\%, proche de celle observée dans l'ensemble du pays (Mintsa Ndong et al., 2009). Le risque de développer une toxoplasmose cérébrale dans cette population rurale serait donc identique à celui des populations urbaines.

Notre étude suggère que la prévalence n'aurait pas évolué en 20 ans, la transmission resterait stable et équivalente à celle observée en milieu urbain. Le mode de contamination serait tellurique et interviendrait dans les dix premières années de vie. La prémunition quasi 
complète de la population des femmes en âge de procréer réduirait le risque de toxoplasmose congénitale. Enfin, le risque potentiel de toxoplasmose cérébrale au cours du SIDA est présent dans cette population.

\section{REMERCIEMENTS}

$\mathrm{N}$ ous remercions Mmes Hélène Tiga, Léontine Moudemba et MM. Ludger Lewobo et Justice Mayombo, personnels de santé de la base CIRMF de Dienga pour leur collaboration.

\section{REFERENCES}

Beauvais B., Garin Y., Languillat G. \& Larivière M. La toxoplasmose au Gabon oriental, résultat d'une enquête sérologique. Bulletin de la Société de Pathologie Exotique, 1978, 71, 172-181.

Billiault X., Collet M., Dupont A. \& Lefevre S. Toxoplasmose chez la femme enceinte dans la province du Haut-Ogooué (Gabon). Bulletin de la Société de Pathologie Exotique, 1987, 80, 74-83.

Carme B., Bissuel F. \& Ajzenberg D. Severe acquired toxoplasmosis in immunocompetent adult patients in French Guiana. Journal of Clinical Microbiology, 2002, 40, 40374044

Carme B. \& Demar-Pierre M. La toxoplasmose en Guyane française : particularité (néo)tropicale d'une parasitose cosmopolite. Médecine Tropicale, 2006, 66, 495-503.

Duong T.H., Martz M., Rondi M.L., Richard-Lenoble D. \& Kombila M. Toxoplasmose au Gabon, résultat d'une enquête séroépidémiologique. Bulletin de la Société de Pathologie Exotique, 1992a, 85, 368-373.

Duong T.H., Dufillot D., Martz M., Richard-Lenoble D. \& Kombila M. Étude séro-épidémiologique de la toxoplasmose à Libreville, Gabon. Annales de la Société Belge de Médecine Tropicale, 1992b, 72, 289-293.

FAn C.K., Hung C.C., Su K.E., Sung F.C., Chiou H.Y., GIL V. et al. Seroprevalence of Toxoplasma gondii infection among pre-schoolchildren aged 1-5 years in the Democratic Republic of Sao Tome and Principe, Western Africa. Transactions of Royal Society of Tropical Medecine and Hygiene, 2006, 100, 446-449.

Mintsa-Ndong A., Caron M., Plantier J.C., Makuwa M., Le Helo S., Courgnaud V., Roques P. \& Kazandji M. High HIV type 1 prevalence and wide genetic diversity with dominance of recombinant strains but low level of antiretroviral drug-resistance mutations in untreated patients in northeast Gabon, Central Africa. AIDS Research and Human Retroviruses. 2009, 25, 411-418.

Negash T., Tilahun G. \& Medhin G. Seroprevalence of Toxoplasma gondii in Nazaret town, Ethiopia. East African Journal of Public Health, 2008, 5, 211-214.
Ngoubangoye B. Étude comparative de la distribution de Toxoplasma gondii parmi les populations humaines et animales, et contribution à l'étude de la virulence des souches en circulation dans un village de la forêt équatoriale : Dienga au Sud-est du Gabon. Thèse, École inter-états de Médecine vétérinaire, Université Cheikh Anta Diop de Dakar, 2007, p. 113.

Okome-Nkoumou M., Mbounja-Loclo M.E. \& Kombila M. Panorama des affections opportunistes au cours de l'infection par le VIH à Libreville, Gabon. Cahiers Santé, 2000, 10, 329-337.

Okоme-Nkoumou M., Boguikouma J.B. \& Kombila M. Les maladies opportunistes de l'infection par le VIH à l'hôpital Fondation Jeanne Ebori de Libreville, Gabon. Médecine Tropicale, 2006, 66, 167-171.

Uneke C.J., Duhuinska D.D., Ngwu B.A. \& Njoku M.O. Seroprevalence of Toxoplasma gondii infection in Kwal, a rural district of Plateau-Nigeria. African Journal Medecine and Medical Sciences, 2007, 36, 109-113.

Reçu le 30 mars 2009 Accepté le 7 juillet 2009 